\title{
Extremal Solutions for Caputo Conformable Differential Equations with $p$-Laplacian Operator and Integral Boundary Condition
}

\author{
Zhongqi Peng $\mathbb{D},{ }^{1}$ Yuan Li $\mathbb{D}^{1},{ }^{1}$ Qi Zhang $\mathbb{D},{ }^{1}$ and Yimin Xue $\mathbb{D}^{2}$ \\ ${ }^{1}$ School of Science, Shenyang University of Technology, Shenyang 110870, China \\ ${ }^{2}$ School of Mathematics and Statistics, Xuzhou University of Technology, Xuzhou 221018, China \\ Correspondence should be addressed to Yuan Li; syliyy@163.com
}

Received 16 August 2021; Accepted 6 October 2021; Published 25 October 2021

Academic Editor: Ning Wang

Copyright (C) 2021 Zhongqi Peng et al. This is an open access article distributed under the Creative Commons Attribution License, which permits unrestricted use, distribution, and reproduction in any medium, provided the original work is properly cited.

The Caputo conformable derivative is a new Caputo-type fractional differential operator generated by conformable derivatives. In this paper, using Banach fixed point theorem, we obtain the uniqueness of the solution of nonlinear and linear Cauchy problem with the conformable derivatives in the Caputo setting, respectively. We also establish two comparison principles and prove the extremal solutions for nonlinear fractional $p$-Laplacian differential system with Caputo conformable derivatives by utilizing the monotone iterative technique. An example is given to verify the validity of the results.

\section{Introduction}

In recent years, fractional calculus has been widely developed in pure mathematics and applied mathematics [1-7]. The characteristic of fractional calculus is that there are many different fractional derivatives or integrals, like Riemann-Liouville (RL), Caputo, Hadamard, Caputo-Hadamard types, and so on [1, 2, 8, 9]. So, the scholars have the opportunity to choose the most appropriate operators to describe complex problems in the real world. We recall some definitions from the traditional fractional calculus $[1,2]$.

The left RL fractional integral of order $\beta>0$ is given by

$$
{ }_{a}^{R} I^{\beta} f(t)=\frac{1}{\Gamma(\beta)} \int_{a}^{b}(t-s)^{\beta-1} f(s) \mathrm{d} s .
$$

The left RL fractional derivative of order $\beta>0$ is defined as

$$
{ }_{a} D^{\beta} f(t)=\left(\frac{\mathrm{d}}{\mathrm{d} t}\right)^{n}{ }_{a} I^{n-\beta} f(t)=\frac{(\mathrm{d} / \mathrm{d} t)^{n}}{\Gamma(n-\beta)} \int_{a}^{t}(t-s)^{n-\beta-1} f(s) \mathrm{d} s .
$$

The left Caputo fractional derivative of order $\beta>0$ is given by

$$
{ }_{a}^{C} D^{\beta} f(t)={ }_{a} I^{n-\beta} f^{(n)}(t)=\frac{1}{\Gamma(n-\beta)} \int_{a}^{t}(t-s)^{n-\beta-1} f^{(n)}(s) \mathrm{d} s .
$$

However, some basic properties such as product rule and chain rule are not valid for the RL and Caputo-type fractional derivatives. In 2014, Khalil et al. [10] defined a new fractional differential operator named the conformable derivative which satisfies the product rule and some other properties. In 2015, Abdeljawad [11] defined the left conformable integral ${ }_{a} I^{\alpha}$ and derivative ${ }_{a} T^{\alpha}$ as

$$
\begin{aligned}
a^{\alpha} I^{\alpha} f(t) & =\frac{1}{\Gamma(\alpha)} \int_{a}^{b}(s-a)^{\alpha-1} f(s) \mathrm{d} s, \\
{ }_{a} T^{\alpha} f(t) & =\lim _{\varepsilon \longrightarrow 0} \frac{f\left(t+\varepsilon(t-a)^{1-\alpha}\right)-f(t)}{\varepsilon},
\end{aligned}
$$

where $\alpha \in(0,1], t>0, f:[a,+\infty) \longrightarrow \mathbb{R}$. If $f$ is differentiable, then ${ }_{a} T^{\alpha} f(t)=(t-a)^{1-\alpha} f^{\prime}(t)$. 
In 2017, Jarad et al. [12] established the conformable calculus in both RL and Caputo setting based on the work of Abdeljawad.

The left RL conformable integral of order $\beta \in \mathbb{C}, \operatorname{Re}(\beta)>0$, is given by

$$
{ }_{a}^{\beta} I^{\alpha} f(t)=\frac{1}{\Gamma(\beta)} \int_{a}^{t}\left(\frac{(t-a)^{\alpha}-(s-a)^{\alpha}}{\alpha}\right)^{\beta-1} f(s) \frac{\mathrm{d} s}{(s-a)^{1-\alpha}} .
$$

The fractional integral (5) coincides with the traditional RL fractional integral (1) if $\alpha=1$.

Let $I_{\alpha}([a, b])=\left\{f:[a, b] \longrightarrow \mathbb{R} ; f(t)={ }_{a} I^{\alpha} \psi(t)+f(a)\right.$ for some $\left.\psi \in L_{\alpha}(a)\right\}, \quad$ where $\quad L_{\alpha}(a)=\{\psi:[a, b] \longrightarrow \mathbb{R}$; ${ }_{a} I^{\alpha} \psi(t)$ exists for any $\left.t \in[a, b]\right\}$. If $n \in \mathbb{N}^{+}, \quad f \in C_{\alpha, a}^{n}$ $[a, b]=\left\{f:[a, b] \longrightarrow \mathbb{R} ;{ }_{a}^{n-1} T^{\alpha} f \in I_{\alpha}([a, b])\right\}$, the left conformable derivative of order $\beta \in \mathbb{C}, \operatorname{Re}(\beta)>0$, in the Caputo setting is defined by

$$
\begin{aligned}
{ }_{a}^{C \beta} D^{\alpha} f(t)= & { }_{a}^{n-\beta} I^{\alpha}\left({ }_{a}^{n} T^{\alpha} f(t)\right) \\
= & \frac{1}{\Gamma(n-\beta)} \int_{a}^{t}\left(\frac{(t-a)^{\alpha}-(s-a)^{\alpha}}{\alpha}\right)^{n-\beta-1} \\
& \frac{{ }_{a}^{n} T^{\alpha} f(s)}{(s-a)^{1-\alpha}} \mathrm{d} s,
\end{aligned}
$$

where

$$
n=[\beta]+1, \quad{ }_{a}^{n} T^{\alpha}=\underbrace{{ }_{a}^{T_{a}^{\alpha} T^{\alpha} \ldots a} T^{\alpha}}_{n \text { times }}
$$

${ }_{a} T^{\alpha} f(t)=(t-a)^{1-\alpha} f^{\prime}(t)$. The fractional derivative (6) coincides with the traditional Caputo fractional derivative (3) if $\alpha=1$. Readers can see $[13,14]$ for more details.

It is well known that the monotone iterative technique coupled with the method of upper and lower solutions is an effective mechanism to obtain extremal solutions for nonlinear problems [15]. By using this method, scholars have studied the periodic boundary value problems (BVPs) [16-24], anti-periodic BVPs [25-27], and integral BVPs [28, 29] of integer-order differential equations. Later, this method was widely used to study the initial value problems, periodic BVPs or integral BVPs of RL and Caputo fractional differential equations [30-35].

Mathematical modeling of the real world in physics and mechanical and dynamical systems often involves the $p$-Laplacian operator. In order to study the turbulent flow in a porous medium, Lejbenson [36] introduced the model of differential equation with the $p$-Laplacian operator. Many results about the fractional differential equations with the p-Laplacian operator were also studied [37-39]. However, the Caputo conformable fractional differential equations with the $p$-Laplacian operator have not been considered.

In [37], Liu et al. studied the following problem:

$$
\left\{\begin{array}{l}
D_{0^{+}}^{\beta}\left(\phi_{p}\left({ }^{C} D_{0^{+}}^{\alpha} x(t)\right)\right)=h\left(t, x(t),{ }^{C} D_{0^{+}}^{\alpha} x(t)\right), \quad t \in(0,1), \\
{ }^{C} D_{0^{+}}^{\alpha} x(0)=x^{\prime}(0)=0, \\
x(1)=r_{1} x(\eta),{ }^{C} D_{0^{+}}^{\alpha} x(1)=r_{2}{ }^{C} D_{0^{+}}^{\alpha} x(\xi),
\end{array}\right.
$$

where $1<\alpha, \beta \leq 2, r_{1}, r_{2} \geq 0, h \in C([0,1] \times t[0,+\infty) n \times q(-\infty$, $0] h,[0,+\infty))$, and $\phi_{p}(s)$ is the $p$-Laplacian operator. The extremal solutions were obtained under the assumption that $h\left(t, w_{1}, z_{1}\right) \leq h\left(t, w_{2}, z_{2}\right), \quad$ for $0 \leq w_{1}<w_{2}, z_{1}>z_{2} \geq 0$.

Inspired by the above work, we study the nonlinear fractional $p$-Laplacian differential system involving the Caputo conformable derivatives as follows:

$$
\left\{\begin{array}{l}
{ }_{a}^{C \beta} D^{\alpha}\left(\phi_{p}\left({ }_{a}^{C \gamma} D^{\alpha} x(t)\right)\right)=h\left(t, x(t),{ }_{a}^{C \gamma} D^{\alpha} x(t)\right), \quad t \in[a, b], \\
{ }_{a}^{k} T^{\alpha} \phi_{p}\left({ }_{a}^{C \gamma} D^{\alpha} x(a)\right)=b_{k}, x(a)=\int_{a}^{b} w(s, x(s)) \mathrm{d} s+\rho,
\end{array}\right.
$$

where $\quad n-1<\beta \leq n, n=[\beta]+1,0<\gamma, \alpha \leq 1, \quad \rho \geq 0$, $h \in C\left([a, b] \times \mathbb{R}^{2}, \mathbb{R}\right), \quad w \in C([a, b] \times \mathbb{R}, \mathbb{R}), \quad b_{k}(k=0,1$, $\ldots, n-1)$ are real numbers, $\phi_{p}(s)=|s|^{p-2} s(p>1)$ is the $p$-Laplacian operator, $\left(\phi_{p}\right)^{-1}=\phi_{q},(1 / p)+(1 / q)=1$, and ${ }_{a}^{C \delta} D^{\alpha}$ is Caputo conformable derivative with order $\delta(=\beta, \gamma)$.

To obtain the extremal solutions of problem (9), we need consider the nonlinear Cauchy problem

$$
\begin{aligned}
& { }_{a}^{C \beta} D^{\alpha} z(t)=g(t, z(t)), \\
& { }_{a}^{k} T^{\alpha} z(a)=b_{k}, \quad t \in(a, b],
\end{aligned}
$$

and the linear Cauchy problem

$$
\begin{aligned}
& { }_{a}^{C \beta} D^{\alpha} z(t)=\sigma(t)-\lambda z(t), \\
& { }_{a}^{k} T^{\alpha} z(a)=b_{k}, \quad t \in[a, b] .
\end{aligned}
$$

The main contributions of this paper are as follows:

(i) We obtain the unique solution to problem (10) and construct the approximate solutions to problem (11) in terms of Mittag-Leffler function. The corresponding results of problem (10) and problem (11) can be seen as a generalization of Theorem 3.25 and Theorem 4.3 in [1], respectively.

(ii) Based on two comparison principles, we obtain the extremal solutions to problem (9) by using the monotone iterative technique. Different from [37], the restrictive condition of function $h$ is no longer needed in this paper.

The remainder of this paper is organized as follows. In Section 2, we give some preliminaries and define some special spaces. In Section 3, we show the uniqueness of the solution for linear and nonlinear Cauchy problems. In Section 4, two comparison principles are established. In Section 5, the extremal solutions for problem (9) are obtained. In Section 6, a numerical example is given.

\section{Preliminaries}

In this section, we introduce some definitions and lemmas to be used in the sequel.

Let $C^{n}(J, \mathbb{R})$ be a Banach space of all $n$-order continuously differentiable functions on $J=[a, b]$. For $n-1<\beta \leq n, 0<\gamma, \alpha \leq 1$, and $0 \leq \tau<1$ such that $\tau \leq \beta-k$, we define the spaces $C_{\gamma}(J), C_{\alpha, \tau}(J), C_{T}^{n}(J), C_{T, \tau}^{n}(J)$, and $C_{T, \tau}^{\beta, n}(J)$ as follows: 


$$
C_{\gamma}(J)=\left\{f: f(t) \in C(J),{ }_{a}^{C \gamma} D^{\alpha} f(J) \in C(J)\right\},
$$

under the norm $\|f\|_{\gamma}=\|f\|_{C}+\left\|_{a}^{C \gamma} D^{\alpha} f\right\|_{C}$, where $\|f\|_{C}=\max _{t \in j}|f(t)|$ and $\left\|_{a}^{C \gamma} D^{\alpha} f\right\|_{C}=\left.\max _{t \in J}\right|_{a} ^{C \gamma} D^{\alpha} f(t) \mid$.

$$
\begin{aligned}
& C_{\alpha, \tau}(J)=\left\{f(t) \in C(a, b]:\left(\frac{(t-a)^{\alpha}}{\alpha}\right)^{\tau} f(t) \in C(J)\right\}, \\
& C_{\alpha, 0}(J)=C(J),
\end{aligned}
$$

under the norm

$$
\begin{aligned}
& \|f\|_{C_{\alpha, \tau}}=\left\|\left(\frac{(t-a)^{\alpha}}{\alpha}\right)^{\tau} f\right\|_{C}=\max _{t \in J}\left|\left(\frac{(t-a)^{\alpha}}{\alpha}\right)^{\tau} f(t)\right|, \\
& C_{T}^{n}(J)=\left\{f(t) \in C(J):{ }_{a}^{n} T^{\alpha} f(t) \in C(J)\right\},
\end{aligned}
$$

under the norm

$$
\begin{aligned}
\|f\|_{C_{T}^{n}} & =\sum_{k=0}^{n}\left\|_{a}^{n} T^{\alpha} f\right\|_{C}=\left.\sum_{k=0}^{n} \max _{t \in J}\right|_{a} ^{n} T^{\alpha} f(t) \mid, \\
C_{T, \tau}^{n}(J) & =\left\{f(t) \in C(J):{ }_{a}^{n-1} T^{\alpha} f(t) \in C(J),{ }_{a}^{n} T^{\alpha} f(t) \in C_{\alpha, \tau}(J)\right\}, \\
C_{T, \tau}^{\beta, n}(J) & =\left\{f(t) \in C_{T}^{n-1}(J):{ }_{a}^{C \beta} D^{\alpha} f(t) \in C_{\alpha, \tau}(J)\right\} .
\end{aligned}
$$

For convenience, we present the following assumptions:

$\left(\mathbf{H}_{1}\right)$ For $t \in(a, b], z_{1}, z_{2} \in C_{T}^{n-1}(J)$, assume that function $g$ satisfies

$$
\left|g\left(t, z_{1}(t)\right)-g\left(t, z_{2}(t)\right)\right| \leq M\left|z_{1}(t)-z_{2}(t)\right|, \quad M \geq 0 .
$$

$\left(\mathbf{H}_{2}\right) \quad$ Assume that $x_{0}(t) \leq y_{0}(t), t \in J$, where $x_{0}(t), y_{0}(t) \in C_{\gamma}(J)$ are lower and upper solutions of (9), respectively.

$\left(\mathbf{H}_{3}\right)$ Assume that a constant $\lambda \leq 0$ such that

$$
\begin{gathered}
h\left(t, y(t),{ }_{a}^{C \gamma} D^{\alpha} y(t)\right)-h\left(t, x(t),{ }_{a}^{C \gamma} D^{\alpha} x(t)\right) \\
\geq-\lambda\left(\phi_{p}\left({ }_{a}^{C \gamma} D^{\alpha} y(t)\right)-\phi_{p}\left({ }_{a}^{C \gamma} D^{\alpha} x(t)\right)\right),
\end{gathered}
$$

where $x_{0}(t) \leq x(t) \leq y(t) \leq y_{0}(t), t \in J$.

$\left(\mathbf{H}_{4}\right)$ Assume that a constant $0 \leq \eta<(1 /(b-a))$ such that

$$
w(t, y(t))-w(t, x(t)) \geq \eta(y(t)-x(t)),
$$

where $x_{0}(t) \leq x(t) \leq y(t) \leq y_{0}(t), t \in J$.

Definition 1. The function $x_{0}(t) \in C_{\gamma}(J)$ satisfying $\phi_{p}$ $\left({ }_{a}^{C \gamma} D^{\alpha} x_{0}(t)\right) \in C_{T}^{n}(J)$ is a lower solution of problem (9) if it satisfies

$$
\left\{\begin{array}{l}
{ }_{a}^{C \beta} D^{\alpha}\left(\phi_{p}\left({ }_{a}^{C \gamma} D^{\alpha} x_{0}(t)\right)\right) \leq h\left(t, x_{0}(t),{ }_{a}^{C \gamma} D^{\alpha} x_{0}(t)\right), \quad t \in J, \\
{ }_{a}^{k} T^{\alpha} \phi_{p}\left({ }_{a}^{C \gamma} D^{\alpha} x_{0}(a)\right) \leq b_{k}, \\
x(a) \leq \int_{a}^{b} w\left(s, x_{0}(s)\right) \mathrm{d} s+\rho .
\end{array}\right.
$$

The function $y_{0}(t) \in C_{\gamma}(J)$ satisfying $\phi_{p}\left({ }_{a}^{C \gamma} D^{\alpha} y_{0}(t)\right) \epsilon$ $C_{T}^{n}(J)$ is an upper solution of problem (9) if the above inequalities are reversed.

Lemma 1 (see [12]). For $\alpha>0$, the space $C_{\alpha, a}^{n}(J)$ consists of those and only those functions which are represented in the form

$$
\begin{aligned}
f(t)= & \frac{1}{(n-1) !} \int_{a}^{t}\left(\frac{(t-a)^{\alpha}-(s-a)^{\alpha}}{\alpha}\right)^{n-1} \frac{\psi(s) \mathrm{d} s}{(s-a)^{1-\alpha}} \\
& +\sum_{k=0}^{n-1} \frac{a^{k} T^{\alpha} f(a)}{k !} \frac{(x-a)^{\alpha k}}{\alpha^{k}}
\end{aligned}
$$

where $\psi(t)={ }_{a}^{n} T^{\alpha} f(t)$ and $\psi(t) \in L_{\alpha}(a)$.

Lemma 2 (see [12]). Let $f \in C_{\alpha, a}^{n}(J), \beta \in \mathbb{C}$. Then,

$$
{ }_{a}^{\beta} I^{\alpha}\left({ }_{a}^{C \beta} D^{\alpha} f(t)\right)=f(t)-\sum_{k=0}^{n-1} \frac{a^{k} T^{\alpha} f(a)(t-a)^{\alpha k}}{\alpha^{k} k !} .
$$


Remark 1. Lemma 1 still holds if we replace the space $C_{\alpha, a}^{n}(J)$ with $C_{T, \tau}^{n}(J)$. In such case, $\psi(t)={ }_{a}^{n} T^{\alpha} f(t)$ and $\psi(t) \in C_{\alpha, \tau}(J)$. In particular, $\psi(t) \in C(J)$ when $\tau=0$. Lemma 2 is also valid for $f \in C_{T}^{n}(J)$.

Lemma 3 (see [12]). Let $\operatorname{Re}(\beta)>0, \operatorname{Re}(\gamma)>0,0<m<\operatorname{Re}(\beta)$, $m \in \mathbb{N}$. Then,
(a) ${ }_{a}^{\beta} I^{\alpha}\left({ }_{a}^{\gamma} I^{\alpha}\right) f(t)={ }_{a}^{\beta+\gamma} I^{\alpha} f(t)$.
(b) ${ }_{a}^{\beta} I^{\alpha}(t-a)^{\alpha(\gamma-1)}=\left(1 / \alpha^{\beta}\right)$ $(t-a)^{\alpha(\beta+\gamma-1)}$.
$(\Gamma(\gamma) / \Gamma(\beta+\gamma))$
(c) ${ }_{a}^{m} T^{\alpha}\left({ }_{a}^{\beta} I^{\alpha} f(t)\right)={ }_{a}^{\beta-m} I^{\alpha} f(t)$.

Lemma 4 (see [1]) (Banach fixed point theorem). Let $(U, d)$ be a nonempty complete metric space, let $0 \leq \rho<1$, and let $A: U \longrightarrow U$ be the map such that, for every $x, y \in U$, the relation

$$
d(A x, A y) \leq \rho d(x, y),
$$

holds. Then, the operator A has a unique fixed point $x^{*} \in U$. Moreover, if $A^{k}(k \in \mathbb{N})$ is the sequence of operators defined by

$$
\begin{aligned}
& A^{1}=A, \\
& A^{k}=A A^{k-1}(k \in \mathbb{N} \backslash\{1\}),
\end{aligned}
$$

then for any $x_{0} \in U$, the sequence $\left\{A^{k} x_{0}\right\}_{k=1}^{\infty}$ converges to the above fixed point $x^{*}$.

\section{The Unique Solution to the Nonlinear and Linear Cauchy Problems}

In this section, we first consider the unique solution of nonlinear Cauchy problem (10) and linear Cauchy problem (11), where the function $g:(a, b] \times \mathbb{R} \longrightarrow \mathbb{R}$ such that $g \in C_{\alpha, \tau}(J)$.

Let $z(t) \in C_{T}^{n-1}(J)$, and by Lemma 2 and the initial value condition ${ }_{a}^{k} T^{\alpha} z(t)=b_{k}$, problem (10) can be reduced to the Volterra-type integral equation

$$
\begin{aligned}
z(t)= & \sum_{j=0}^{n-1} \frac{b_{j}(t-a)^{\alpha j}}{\alpha^{j} j !}+\frac{1}{\Gamma(\beta)} \int_{a}^{t} K^{\beta-1}(t, s) g(s, z(s)) \\
& \frac{\mathrm{d} s}{(s-a)^{1-\alpha}},
\end{aligned}
$$

where $\quad K(t, s)=\left(\left((t-a)^{\alpha}-(s-a)^{\alpha}\right) / \alpha\right), a \leq s \leq t \leq b$. Denoting $z_{0}=\sum_{j=0}^{n-1}\left(b_{j}(t-a)^{\alpha j} / \alpha^{j} j !\right)$, equation (24) can be rewritten as $z(t)=(A z)(t)$, where

$$
(A z)(t)=z_{0}(t)+\frac{1}{\Gamma(\beta)} \int_{a}^{t} K^{\beta-1}(t, s) g(s, z(s)) \frac{\mathrm{d} s}{(s-a)^{1-\alpha}} .
$$

Theorem 1. If $\left(\mathrm{H}_{1}\right)$ holds, there exists a unique solution $z(t) \in C_{T, \tau}^{\beta, n-1}(J)$ for problem (10).

Proof. First, we choose $t_{1}\left(a<t_{1}<b\right)$ such that

$$
\sum_{k=0}^{n-1} \frac{M}{\Gamma(\beta-k+1)}\left(\frac{\left(t_{1}-a\right)^{\alpha}}{\alpha}\right)^{\beta-k}<1,
$$

and prove that (10) has a unique solution $z(t) \in C_{T}^{n-1}\left[a, t_{1}\right]$. Applying the operator ${ }_{a}^{k} T^{\alpha}$ to both sides of (25), by Lemma 3 (c), we can get

$$
\begin{gathered}
{ }_{a}^{k} T^{\alpha}(A z(t))={ }_{a}^{k} T^{\alpha} z_{0}(t)+\frac{1}{\Gamma(\beta-k)} \int_{a}^{t} K^{\beta-k-1} \\
(t, s) \frac{g(s, z(s)) \mathrm{d} s}{(s-a)^{1-\alpha}}
\end{gathered}
$$

where ${ }_{a}^{k} T^{\alpha} z_{0}(t)=\sum_{j=k}^{n-1}\left(b_{j}(t-a)^{(j-k)} / \alpha^{j-k}(j-k) !\right)$. It is obvious that ${ }_{a}^{k} T^{\alpha} z_{0}(t)$ is continuous on $\left[a, t_{1}\right]$. Furthermore, for $0 \leq \tau<\beta-k$ and $g \in C_{\alpha, \tau}(J)$, we get by (14) that

$$
\begin{aligned}
||_{a}^{\beta-k} I^{\alpha} g \mid & =\left|\frac{1}{\Gamma(\beta-k)} \int_{a}^{t} K^{\beta-k-1}(t, s) g(s, z(s)) \frac{\mathrm{d} s}{(s-a)^{1-\alpha}}\right| \\
& \leq \frac{1}{\Gamma(\beta-k)} \int_{a}^{t}\left(\frac{(t-a)^{\alpha}-(s-a)^{\alpha}}{\alpha}\right)^{\beta-k-1}|g(s, z(s))| \frac{\mathrm{d} s}{(s-a)^{1-\alpha}} \\
& \leq \frac{\|g\|_{C_{\alpha, \tau}\left[a, t_{1}\right]}}{\Gamma(\beta-k)} \int_{a}^{t}\left(\frac{(t-a)^{\alpha}-(s-a)^{\alpha}}{\alpha}\right)^{\beta-k-1}\left(\frac{(s-a)^{\alpha}}{\alpha}\right)^{-\tau} \frac{\mathrm{d} s}{(s-a)^{1-\alpha}} \\
& \leq \frac{\Gamma(1-\tau)\|g\|_{C_{\alpha, \tau}\left[a, t_{1}\right]}\left(\frac{\left(t_{1}-a\right)^{\alpha}}{\alpha(\beta-k+1-\tau)}\right)^{\beta-k-\tau},}{\alpha+k}
\end{aligned}
$$


that is,

$$
\left\|\left.\right|_{a} ^{\beta-k} I^{\alpha} g\right\|_{C\left[a, t_{1}\right]} \leq \frac{\Gamma(1-\tau)\|g\|_{C_{\alpha, \tau}\left[a, t_{1}\right]}}{\Gamma(\beta-k+1-\tau)}\left(\frac{\left(t_{1}-a\right)^{\alpha}}{\alpha}\right)^{\beta-k-\tau} .
$$

Inequality (29) implies that the operator ${ }_{a}^{\beta-k} I^{\alpha}$ is bounded from $C_{\alpha, \tau}\left[a, t_{1}\right]$ to $C\left[a, t_{1}\right]$. In particular, if $\tau=0$, then $g \in C(J)$ and ${ }_{a}^{\beta-k} I^{\alpha}$ is bounded from $C\left[a, t_{1}\right]$ to $C\left[a, t_{1}\right]$ such that

$$
\left\|a_{a}^{\beta-k} I^{\alpha} g\right\|_{C\left[a, t_{1}\right]} \leq \frac{\|g\|_{C\left[a, t_{1}\right]}}{\Gamma(\beta-k+1)}\left(\frac{\left(t_{1}-a\right)^{\alpha}}{\alpha}\right)^{\beta-k} .
$$

It follows from (29) and (30) that ${ }_{a}^{k} T^{\alpha}(A z(t))$ is continuous on $\left[a, t_{1}\right]$, that is, $(A z)(t) \in C_{T}^{n-1}\left[a, t_{1}\right]$. By (14), (15), and $\left(H_{1}\right)$, we have

$$
\begin{aligned}
& \sum_{k=0}^{n-1}\left|{ }_{a}^{k} T^{\alpha}\left(A z_{1}(t)\right)-{ }_{a}^{k} T^{\alpha}\left(A z_{2}(t)\right)\right| \\
& \quad=\sum_{k=0}^{n-1}\left|\frac{1}{\Gamma(\beta-k)} \int_{a}^{t} K^{\beta-k-1}(t, s)\left[g\left(s, z_{1}(s)\right)-g\left(s, z_{2}(s)\right)\right] \frac{\mathrm{d} s}{(s-a)^{1-\alpha}}\right| \\
& \quad \leq \sum_{k=0}^{n-1} \frac{1}{\Gamma(\beta-k)} \int_{a}^{t} K^{\beta-k-1}(t, s)\left|g\left(s, z_{1}(s)\right)-g\left(s, z_{2}(s)\right)\right| \frac{\mathrm{d} s}{(s-a)^{1-\alpha}} \\
& \quad \leq \sum_{k=0}^{n-1} \frac{M}{\Gamma(\beta-k)} \int_{a}^{t}\left(\frac{(t-a)^{\alpha}-(s-a)^{\alpha}}{\alpha}\right)^{\beta-k-1}\left|z_{1}(s)-z_{2}(s)\right| \frac{\mathrm{d} s}{(s-a)^{1-\alpha}} \\
& \quad \leq \sum_{k=0}^{n-1} \frac{M\left\|z_{1}-z_{2}\right\|_{C}\left[a, t_{1}\right]}{\Gamma(\beta-k+1)}\left(\frac{\left(t_{1}-a\right)^{\alpha}}{\alpha}\right)^{\beta-k} \\
& \quad \leq \sum_{k=0}^{n-1} \frac{M\left\|z_{1}-z_{2}\right\|_{C_{T}^{n-1}\left[a, t_{1}\right]}}{\Gamma(\beta-k+1)}\left(\frac{\left(t_{1}-a\right)^{\alpha}}{\alpha}\right)^{\beta-k},
\end{aligned}
$$

that is,

$$
\begin{aligned}
\left\|A z_{1}-A z_{2}\right\|_{C_{T}^{n-1}\left[a, t_{1}\right]} & =\sum_{k=0}^{n-1}\|\|_{a}^{k} T^{\alpha}\left(A z_{1}(t)\right)-{ }_{a}^{k} T^{\alpha}\left(A z_{2}(t)\right) \|_{C\left[a, t_{1}\right]} \\
& \leq \sum_{k=0}^{n-1} \frac{M\left\|z_{1}-z_{2}\right\|_{C_{T}^{n-1}\left[a, t_{1}\right]}}{\Gamma(\beta-k+1)}\left(\frac{\left(t_{1}-a\right)^{\alpha}}{\alpha}\right)^{\beta-k} .
\end{aligned}
$$

From Lemma 4 and (26), we get that there exists a unique solution $z_{1}^{*} \in C_{T}^{n-1}\left[a, t_{1}\right]$ to problem (10). Moreover, $z_{1}^{*}(t)$ satisfies

$$
\lim _{i \longrightarrow \infty}\left\|A^{i} z_{g}-z_{1}^{*}\right\|_{C_{T}^{n-1}\left[a, t_{1}\right]}=0,
$$

where $z_{g}(t)$ is any function in $C_{T}^{n-1}\left[a, t_{1}\right]$ and $A^{i} z_{g}(t)=A A^{i-1} z_{g}(t)$. Let $z_{i}(t)=A^{i} z_{g}(t)$; then,

$$
\lim _{i \longrightarrow \infty}\left\|z_{i}(t)-z_{1}^{*}\right\|_{C_{T}^{n-1}\left[a, t_{1}\right]}=0 \text {. }
$$

Next, choose $t_{2}, t_{3}, \ldots, t_{R}$ such that $a=t_{0}<t_{1}<t_{2}<$ $\cdots<t_{R}=b$. Using the same arguments as above, we get that problem (10) has a unique solution $z_{r}^{*} \in C_{T}^{n-1}\left[t_{r-1}, t_{r}\right]$ $(r=1,2, \ldots, R)$. Therefore, (10) has a unique solution $z^{*}=z_{r}^{*} \in C_{T}^{n-1}(J)$.

Finally, we show that the unique solution $z^{*}(t)$ belongs to $C_{T, \tau}^{\beta, n-1}(J)$. By $(15)$ and $\left(H_{1}\right)$, we have

$$
\left\|{ }_{a}^{C \beta} D^{\alpha} z_{i}-{ }_{a}^{C \beta} D^{\alpha} z^{*}\right\|_{C_{\alpha, \tau}(J)}=\left\|g\left(t, z_{i}(t)\right)-g\left(t, z^{*}(t)\right)\right\|_{C_{\alpha, \tau}(J)}
$$

$$
\begin{aligned}
& \leq M\left(\frac{\left(t_{1}-a\right)^{\alpha}}{\alpha}\right)^{\tau}\left\|z_{i}-z^{*}\right\|_{C(J)} \\
& \leq M\left(\frac{\left(t_{1}-a\right)^{\alpha}}{\alpha}\right)^{\tau}\left\|z_{i}-z^{*}\right\|_{C_{T}^{n-1}(J)^{\circ}}
\end{aligned}
$$


Taking the limit as $i \longrightarrow \infty$, we obtain

$$
\lim _{i \longrightarrow \infty}\|\|_{a}^{C \beta} D^{\alpha} z_{i}-{ }_{a}^{C \beta} D^{\alpha} z^{*} \|_{C_{\alpha, \tau}(J)}=0,
$$

which implies that $z^{*}(t) \in C_{T, \tau}^{\beta, n-1}(J)$. This ends the proof.

Remark 2. When $\alpha=1$, the RL conformable integral and Caputo conformable derivative coincide with the traditional RL fractional integral and Caputo derivative, respectively. Hence, the results of Theorem 3.25 in [1] can be seen as the special case of Theorem 1 .

Corollary 1. If $\left(\mathrm{H}_{1}\right)$ holds and the function $g: J \times \mathbb{R} \longrightarrow \mathbb{R}$ satisfies $g \in C(J)$, then Cauchy problem (10) has a unique solution $z(t)$ belonging to $C_{T}^{n-1}(J)$.

Proof. Corollary 1 can be proven by replacing $\tau$ with 0 and using the same argument in Theorem 1.

Theorem 2. If $\sigma(t) \in C(J)$ and $\lambda$ is a constant, then problem (11) has a unique solution $z(t) \in C_{T}^{n-1}(J)$ which is given by

$$
\begin{aligned}
z(t)= & \sum_{j=0}^{n-1} \frac{b_{j}(t-a)^{j \alpha}}{\alpha^{j}} E_{\beta, j+1}\left[\frac{-\lambda(t-a)^{\alpha \beta}}{\alpha^{\beta}}\right] \\
& +\int_{a}^{t} K^{\beta-1}(t, s) E_{\beta, \beta}\left(-\lambda K^{\beta}(t, s)\right) \frac{\sigma(s) \mathrm{d} s}{(s-a)^{1-\alpha}},
\end{aligned}
$$

where $E_{p, q}(\rho)=\sum_{k=0}^{\infty}\left(\rho^{k} / \Gamma(p k+q)\right)$ is the Mittag-Leffler function.
Proof. Clearly $g(t, z(t))=\sigma(t)-\lambda z(t)$ satisfies $\left(H_{1}\right)$. By Corollary 1 , there exists a unique solution $z(t) \in C_{T}^{n-1}(J)$ to problem (11).

Next, we prove that this unique solution is given by (18). By Lemma 2 and the initial value condition ${ }_{a}^{k} T^{\alpha} z(t)=b_{k}$, problem (11) can be reduced to the equation

$$
z(t)=\sum_{j=0}^{n-1} \frac{b_{j}(t-a)^{\alpha j}}{\alpha^{j} j !}+(-\lambda)_{a}^{\beta} I^{\alpha} z(t)+{ }_{a}^{\beta} I^{\alpha} \sigma(t) .
$$

We apply the successive approximations method to solve equation (38) by taking $z_{0}(t)=\sum_{j=0}^{n-1} b_{j}(t-a)^{\alpha j} / \alpha^{j} j$ ! and

$$
z_{m}(t)=z_{0}(t)+(-\lambda)_{a}^{\beta} I^{\alpha} z_{m-1}(t)+{ }_{a}^{\beta} I^{\alpha} \sigma(t), \quad m=1,2, \ldots
$$

By Lemma 3 (b), for $m=1$, we have

$$
\begin{aligned}
z_{1}(t) & =z_{0}(t)+(-\lambda)_{a}^{\beta} I^{\alpha} z_{0}(t)+{ }_{a}^{\beta} I^{\alpha} \sigma(t) \\
& =z_{0}(t)+\sum_{j=0}^{n-1} \frac{(-\lambda) b_{j} \beta}{\alpha^{j} j !} I^{\alpha}(t-a)^{\alpha j}+{ }_{a}^{\beta} I^{\alpha} \sigma(t) \\
& =z_{0}(t)+\sum_{j=0}^{n-1} \frac{(-\lambda) b_{j}(t-a)^{\alpha(\beta+j)}}{\alpha^{\beta+j} \Gamma(\beta+j+1)}+{ }_{a}^{\beta} I^{\alpha} \sigma(t) .
\end{aligned}
$$

By (a) and (b) of Lemma 3, for $m=2$,

$$
\begin{aligned}
z_{2}(t)= & z_{0}(t)+(-\lambda)_{a}^{\beta} I^{\alpha} z_{1}(t)+{ }_{a}^{\beta} I^{\alpha} \sigma(t) \\
= & z_{0}(t)+(-\lambda)_{a}^{\beta} I^{\alpha}\left[z_{0}(t)+(-\lambda)_{a}^{\beta} I^{\alpha} z_{0}(t)+{ }_{a}^{\beta} I^{\alpha} \sigma(t)\right]+{ }_{a}^{\beta} I^{\alpha} \sigma(t) \\
= & z_{0}(t)+(-\lambda)_{a}^{\beta} I^{\alpha} z_{0}(t)+(-\lambda)_{a}^{22 \beta} I^{\alpha} z_{0}(t)+(-\lambda)_{a}^{2 \beta} I^{\alpha} \sigma(t)+{ }_{a}^{\beta} I^{\alpha} \sigma(t) \\
= & z_{0}(t)+\sum_{j=0}^{n-1} \frac{(-\lambda) b_{j}(t-a)^{\alpha(\beta+j)}}{\alpha^{\beta+j} \Gamma(\beta+j+1)}+\sum_{j=0}^{n-1} \frac{(-\lambda)^{2} b_{j}(t-a)^{\alpha(2 \beta+j)}}{\alpha^{2 \beta+j} \Gamma(2 \beta+j+1)} \\
& +(-\lambda)_{a}^{2 \beta} I^{\alpha} \sigma(t)+{ }_{a}^{\beta} I^{\alpha} \sigma(t) .
\end{aligned}
$$

Continuing this process, we have

$$
\begin{aligned}
z_{m}(t)= & \sum_{j=0}^{n-1} \frac{b_{j}(t-a)^{j \alpha}}{\alpha^{j}} \sum_{r=0}^{m} \frac{(-\lambda)^{r}(t-a)^{r \alpha \beta}}{\alpha^{r \beta} \Gamma(r \beta+j+1)}+\sum_{r=1}^{m}(-\lambda)_{a}^{r-1 r \beta} I^{\alpha} \sigma(t) \\
= & \sum_{j=0}^{n-1} \frac{b_{j}(t-a)^{j \alpha}}{\alpha^{j}} \sum_{r=0}^{m} \frac{(-\lambda)^{r}(t-a)^{r \alpha \beta}}{\alpha^{r \beta} \Gamma(r \beta+j+1)} \\
& +\int_{a}^{t} K^{\beta-1}(t, s) \sum_{r=0}^{m} \frac{(-\lambda)^{r} K^{r \beta}(t, s)}{\Gamma((r+1) \beta)} \frac{\sigma(s) \mathrm{d} s}{(s-a)^{1-\alpha}} .
\end{aligned}
$$

Taking the limit as $m \longrightarrow \infty$ and according to the definition of Mittag-Leffler function, we get formula (37). This ends the proof.

Remark 3. If $\alpha=1$, we can get the results of Theorem 4.3 in [1].

\section{Comparison Principles}

In this section, two comparison principles which will be used in the next section are established. 
Lemma 5. Let $\eta \neq(1 / b-a)$ and $y(t) \in C(J)$. Then, the is equivalent to following problem:

$$
\left\{\begin{array}{l}
{ }_{a}^{C \gamma} D^{\alpha} x(t)=y(t), \quad t \in J \\
x(a)=\eta \int_{a}^{b} x(s) \mathrm{d} s+\rho
\end{array}\right.
$$

$$
x(t)=\int_{a}^{b} G(t, s) y(s) \frac{\mathrm{d} s}{(s-a)^{1-\alpha}}+\frac{\rho}{1-\eta(b-a)},
$$

where

$$
G(t, s)=\frac{1}{\xi} \begin{cases}\frac{[1-\eta(b-a)] \gamma(b-a)^{\alpha-1} K^{\gamma-1}(t, s)+\eta K^{\gamma}(b, s)}{1-\eta(b-a)}, & a \leq s \leq t \leq b, \\ \frac{\eta K^{\gamma}(b, s)}{1-\eta(b-a)}, & a \leq t \leq s \leq b, \\ \xi=(b-a)^{\alpha-1} \Gamma(\gamma+1) . & \end{cases}
$$

Proof. For $0<\gamma \leq 1$, by Lemma 2, equation ${ }_{a}^{C \gamma} D^{\alpha} x(t)=y(t)$ can be reduced to

$$
x(t)={ }_{a}^{\gamma} I^{\alpha} y(t) c_{0},
$$

$$
x(t)={ }_{a}^{\gamma} I^{\alpha} y(t)+\eta \int_{a}^{b} x(s) \mathrm{d} s+\rho .
$$

where $c_{0}$ is a constant. By the boundary condition, we easily get $c_{0}=\eta \int_{a}^{b} x(s) \mathrm{d} s+\rho$. Hence,

$$
\begin{aligned}
\Delta & =\int_{a}^{b} \frac{1}{\Gamma(\gamma)} \int_{a}^{t}\left(\frac{(t-a)^{\alpha}-(s-a)^{\alpha}}{\alpha}\right)^{\gamma-1} y(s) \frac{\mathrm{d} s}{(s-a)^{1-\alpha}} \mathrm{d} t+\int_{a}^{b}(\eta \Delta+\rho) \mathrm{d} t \\
& =\int_{a}^{b} \frac{1}{\Gamma(\gamma+1)(b-a)^{\alpha-1}}\left(\frac{(b-a)^{\alpha}-(s-a)^{\alpha}}{\alpha}\right)^{\gamma} \frac{y(s) \mathrm{d} s}{(s-a)^{1-\alpha}}+(\eta \Delta+\rho)(b-a) \\
& =\int_{a}^{b} \frac{K^{\gamma}(b, s) y(s)}{\Gamma(\gamma+1)(b-a)^{\alpha-1}} \frac{\mathrm{d} s}{(s-a)^{1-\alpha}}+(\eta \Delta+\rho)(b-a) .
\end{aligned}
$$

Therefore,

$$
\Delta=\int_{a}^{b} \frac{K^{\gamma}(b, s) y(s)}{[1-\eta(b-a)] \Gamma(\gamma+1)(b-a)^{\alpha-1}} \frac{\mathrm{d} s}{(s-a)^{1-\alpha}}+\frac{\rho(b-a)}{1-\eta(b-a)} .
$$


Substituting (49) into (47), we have

$$
\begin{aligned}
x(t)= & \frac{1}{\Gamma(\gamma)} \int_{a}^{t} K^{\gamma-1}(t, s) y(s) \frac{\mathrm{d} s}{(s-a)^{1-\alpha}}+\frac{\eta \rho(b-a)}{1-\eta(b-a)}+\rho \\
& +\int_{a}^{b} \frac{\eta K^{\gamma}(b, s) y(s)}{[1-\eta(b-a)] \Gamma(\gamma+1)(b-a)^{\alpha-1}} \frac{\mathrm{d} s}{(s-a)^{1-\alpha}} \\
= & \frac{1}{\xi} \int_{a}^{t} \frac{[1-\eta(b-a)] \gamma(b-a)^{\alpha-1} K^{\gamma-1}(t, s)+\eta K^{\gamma}(b, s)}{1-\eta(b-a)} y(s) \frac{\mathrm{d} s}{(s-a)^{1-\alpha}} \\
& +\frac{1}{\xi} \int_{t}^{b} \frac{\eta K^{\gamma}(b, s)}{1-\eta(b-a)} y(s) \frac{\mathrm{d} s}{(s-a)^{1-\alpha}}+\frac{\rho}{1-\eta(b-a)} \\
= & \int_{a}^{b} G(t, s) y(s) \frac{\mathrm{d} s}{(s-a)^{1-\alpha}}+\frac{\rho}{1-\eta(b-a)} .
\end{aligned}
$$

This ends the proof.

Remark 4. For $a \leq t, s \leq b, 0 \leq \eta<(1 /(b-a))$, the function $G(t, s)$ is continuous and nonnegative.

Lemma 6. For $x(t) \in C_{\gamma}(J)$, the following linear problem:

$$
\left\{\begin{array}{l}
{ }_{a}^{C \beta} D^{\alpha}\left(\phi_{p}\left({ }_{a}^{C \gamma} D^{\alpha}(t)\right)\right)=\sigma(t)-\lambda \phi_{p}\left({ }_{a}^{C \gamma} D^{\alpha} x(t)\right), \quad t \in J, \\
{ }_{a}^{k} T^{\alpha} \phi_{p}\left({ }_{a}^{C \gamma} D^{\alpha} x(a)\right)=b_{k}, \\
x(a)=\eta \int_{a}^{b} x(s) \mathrm{d} s+\rho,
\end{array}\right.
$$

has a unique solution.

Proof. Let $z(t)=\phi_{p}\left({ }_{a}^{C \gamma} D^{\alpha} x(t)\right)$. By Theorem 2, the problem

$$
\begin{gathered}
{ }_{a}^{C \beta} D^{\alpha} z(t)=\sigma(t)-\lambda z(t), \\
{ }_{a}^{k} T^{\alpha} z(t)=b_{k}, \quad t \in J
\end{gathered}
$$

has ${ }_{\alpha}$ a unique solution $z(t) \in C_{T^{n}}^{n-1}(J)$, that is, ${ }_{a}^{C \gamma} D^{\alpha} x(t)=\phi_{q}(z(t)) \in C_{T}^{n-1}(J)$. Hence, ${ }_{a}^{C \gamma} D^{\alpha} x(t) \in C(J)$.

By Lemma 5, the following problem

$$
\begin{aligned}
{ }_{a}^{C \gamma} D^{\alpha} x(t) & =\phi_{q}(z(t)), \\
x(a) & =\eta \int_{a}^{b} x(s) \mathrm{d} s+\rho, \quad t \in J,
\end{aligned}
$$

is equivalent to

$$
x(t)=\int_{a}^{b} G(t, s) \phi_{q}(z(s)) \frac{\mathrm{d} s}{(s-a)^{1-\alpha}}+\frac{\rho}{1-\eta(b-a)} .
$$

Considering (52) and (53), we obtain the conclusion that problem (51) has a unique solution which is given by (54).
Based on the above work, we can get the following comparison principles.

Lemma 7. If $\lambda \leq 0$ and $z(t) \in C_{T}^{n-1}(J)$ satisfy the following relation:

$$
\left\{{ }_{a}^{C \beta} D^{\alpha} z(t) \geq-\lambda z(t),{ }_{a}^{k} T^{\alpha} z(a) \geq 0,\right.
$$

then for $t \in J, z(t) \geq 0$.

Proof. Let ${ }_{a}^{C \beta} D^{\alpha} z(t)=p(t)-\lambda z(t),{ }_{a}^{k} T^{\alpha} z(a)=a_{k}$; then, $p(t) \geq 0, a_{k} \geq 0$. From (37), we can see that $z(t) \geq 0$. This ends the proof.

Lemma 8. If $0 \leq \eta<(1 /(b-a))$ and $x(t) \in C_{\gamma}(J)$ satisfy the following relation:

$$
\left\{{ }_{a}^{C \gamma} D^{\alpha} x(t) \geq 0, x(a) \geq \eta \int_{a}^{b} x(s) \mathrm{d} s,\right.
$$

then for $t \in J, x(t) \geq 0$.

Proof. Let ${ }_{a}^{C \gamma} D^{\alpha} x(t)=q(t), x(a)=\int_{a}^{b} \eta x(s) \mathrm{d} s+d$; then, $q(t) \geq 0, d \geq 0$. From (53) and (54), we have that

$$
x(t)=\int_{a}^{b} G(t, s) q(s) \frac{\mathrm{d} s}{(s-a)^{1-\alpha}}+\frac{d}{1-\eta(b-a)},
$$

which implies that $x(t) \geq 0$ due to $G(t, s) \geq 0$. This ends the proof.

\section{Extremal Solutions for Nonlinear System}

The extremal solutions of problem (9) are obtained in this section.

Theorem 3. If $\left(\mathrm{H}_{2}\right)-\left(\mathrm{H}_{4}\right)$ hold, then problem (9) has extremal solutions $x^{*}(t), y^{*}(t)$ in the sector $\left[x_{0}, y_{0}\right]=\left\{x(t) \in C_{\gamma}(J): x_{0}(t) \leq x(t) \leq y_{0}(t), t \in J\right\}$.

Moreover, 


$$
\begin{gathered}
x_{0}(t) \leq x^{*}(t) \leq y^{*}(t) \leq y_{0}(t), \\
{ }_{a}^{C \gamma} D^{\alpha} x_{0}(t) \leq{ }_{a}^{C \gamma} D^{\alpha} x^{*}(t) \leq{ }_{a}^{C \gamma} D^{\alpha} y^{*}(t) \leq{ }_{a}^{C \gamma} D^{\alpha} y_{0}(t) .
\end{gathered}
$$

Proof. For $t \in J, n=1,2, \ldots$, define

$$
\begin{aligned}
& \left\{\begin{array}{l}
{ }_{a}^{C \beta} D^{\alpha}\left(\phi_{p}\left({ }_{a}^{C \gamma} D^{\alpha} x_{n}(t)\right)\right)=h\left(t, x_{n-1}(t),{ }_{a}^{C \gamma} D^{\alpha} x_{n-1}(t)\right)-\lambda\left[\phi_{p}\left({ }_{a}^{C \gamma} D^{\alpha} x_{n}(t)\right)-\phi_{p}\left({ }_{a}^{C \gamma} D^{\alpha} x_{n-1}(t)\right)\right], \\
{ }_{a}^{k} T^{\alpha} \phi_{p}\left({ }_{a}^{C \gamma} D^{\alpha} x_{n}(a)\right)=b_{k}, \\
x_{n}(a)=\int_{a}^{b}\left[w\left(s, x_{n-1}(s)\right)+\eta\left(x_{n}(s)-x_{n-1}(s)\right)\right] \mathrm{d} s+\rho,
\end{array}\right. \\
& \left\{\begin{array}{l}
{ }_{a}^{C \beta} D^{\alpha}\left(\phi_{p}\left({ }_{a}^{C \gamma} D^{\alpha} y_{n}(t)\right)\right)=h\left(t, y_{n-1}(t),{ }_{a}^{C \gamma} D^{\alpha} y_{n-1}(t)\right)-\lambda\left[\phi_{p}\left({ }_{a}^{C \gamma} D^{\alpha} y_{n}(t)\right)-\phi_{p}\left({ }_{a}^{C \gamma} D^{\alpha} y_{n-1}(t)\right)\right], \\
{ }_{a} T^{\alpha} \phi_{p}\left({ }_{a}^{C \gamma} D^{\alpha} y_{n}(a)\right)=b_{k}, \\
y_{n}(a)=\int_{a}^{b}\left[w\left(s, y_{n-1}(s)\right)+\eta\left(y_{n}(s)-y_{n-1}(s)\right)\right] \mathrm{d} s+\rho .
\end{array}\right.
\end{aligned}
$$

By Lemma $6, x_{n}, y_{n}$ are well defined. The proof includes three steps.

Step 1. We prove the monotone property of $\left\{x_{n}\right\}$ and $\left\{y_{n}\right\}$.

Let $r(t)=\phi_{p}\left({ }_{a}^{C \gamma} D^{\alpha} x_{1}(t)\right)-\phi_{p}\left({ }_{a}^{C \gamma} D^{\alpha} x_{0}(t)\right)$, and by $\left(H_{2}\right)$ and (59), we get

$$
\left\{{ }_{a}^{C \beta} D^{\alpha} r(t) \geq-\lambda r(t), \quad t \in J,{ }_{a}^{k} T^{\alpha} r(a) \geq 0 .\right.
$$

From Lemma 7 , we have $r(t) \geq 0$, i.e., $\phi_{p}\left({ }_{a}^{C \gamma} D^{\alpha} x_{1}(t)\right) \geq \phi_{p}\left({ }_{a}^{C \gamma} D^{\alpha} x_{0}(t)\right)$. Moreover,

$$
{ }_{a}^{C \gamma} D^{\alpha} x_{1}(t) \geq{ }_{a}^{C \gamma} D^{\alpha} x_{0}(t)
$$

holds because of the monotone increasing property of $\phi_{p}(s)$.

Let $\tilde{r}(t)=x_{1}(t)-x_{0}(t)$. From $\left(H_{2}\right),\left(H_{4}\right),(59)$, and (62), we have

$$
\left\{\begin{array}{l}
{ }_{a}^{C \gamma} D^{\alpha} \widetilde{r}(t)={ }_{a}^{C \gamma} D^{\alpha} x_{1}(t)-{ }_{a}^{C \gamma} D^{\alpha} x_{0}(t) \geq 0 \\
\widetilde{r}(a) \geq \int_{a}^{b} \eta \widetilde{r}(s) \mathrm{d} s
\end{array}\right.
$$

From Lemma 8, we have $\tilde{r}(t) \geq 0$, i.e. ${ }_{\alpha} x_{1}(t) \geq x_{0}(t)$. The same argument holds that ${ }_{\alpha} a^{\gamma} D^{\alpha} y_{0}(t) \geq{ }_{a}^{C \gamma} D_{\alpha}^{\alpha} y_{1}(t)$, $y_{0}(t) \geq y_{1}(t)$. Let $m(t)=\phi_{p}\left({ }_{a}^{C \gamma} D^{\alpha} y_{1}(t)\right)-\phi_{p}\left({ }_{a}^{C \gamma} D^{\alpha} x_{1}(t)\right)$, and from $\left(H_{3}\right)$, (59), and (60), we get

$$
\begin{aligned}
{ }_{a}^{C \beta} D^{\alpha} m(t)= & h\left(t, y_{0}(t){ }_{a}^{C \gamma} D^{\alpha} y_{0}(t)\right)-h\left(t, x_{0}(t),{ }_{a}^{C \gamma} D^{\alpha} x_{0}(t)\right) \\
& -\lambda\left[\phi_{p}\left({ }_{a}^{C \gamma} D^{\alpha} y_{1}(t)\right)-\phi_{p}\left({ }_{a}^{C \gamma} D^{\alpha} y_{0}(t)\right)\right] \\
& +\lambda\left[\phi_{p}\left({ }_{a}^{C \gamma} D^{\alpha} x_{1}(t)\right)-\phi_{p}\left({ }_{a}^{C \gamma} D^{\alpha} x_{0}(t)\right)\right] \\
\geq & -\lambda m(t), \\
{ }_{a}^{k} T^{\alpha} m(a)= & 0 .
\end{aligned}
$$

From Lemma 7 , we have $m(t) \geq 0$, i.e., $\phi_{p}\left({ }_{a}^{C \gamma} D^{\alpha} y_{1}(t)\right) \geq \phi_{p}\left(a^{C \gamma} D^{\alpha} x_{1}(t)\right)$. Hence,

$$
{ }_{a}^{C \gamma} D^{\alpha} y_{1}(t) \geq{ }_{a}^{C \gamma} D^{\alpha} x_{1}(t) \text {. }
$$

Let $\widetilde{m}(t)=y_{1}-x_{1}$. We get from $(65)$ and $\left(H_{4}\right)$ that

$$
\left\{\begin{array}{l}
{ }_{a}^{C \gamma} D^{\alpha} \widetilde{m}(t)={ }_{a}^{C \gamma} D^{\alpha} y_{1}(t)-{ }_{a}^{C \gamma} D^{\alpha} x_{1}(t) \geq 0, \\
\widetilde{m}(a)=\int_{a}^{b}\left[w\left(s, y_{0}(s)\right)+\eta\left(y_{1}(s)-y_{0}(s)\right)-w\left(s, x_{0}(s)\right)-\eta\left(x_{1}(s)-x_{0}(s)\right)\right] \mathrm{d} s \geq \int_{a}^{b} \eta \widetilde{m}(s) \mathrm{d} s .
\end{array}\right.
$$

From Lemma 8, we have $\widetilde{m}(t) \geq 0$, i.e. $y_{1} \geq x_{\alpha}$. Therefore, $x_{0} \leq x_{1} \leq y_{1} \leq y_{0}$ and ${ }_{a}^{C \gamma} D^{\alpha} x_{0} \leq{ }_{a}^{C \gamma} D^{\alpha} x_{1} \leq{ }_{a}^{C \gamma} D^{\alpha} y_{1} \leq{ }_{a}^{C \gamma} D^{\alpha} y_{0}$.
Next we prove $x_{1}(t), y_{1}(t)$ are lower and upper solutions of (9), respectively. From $\left(H_{3}\right),\left(H_{4}\right)$, and (59), we have 


$$
\begin{aligned}
{ }_{a}^{C \beta} D^{\alpha}\left(\phi_{p}\left({ }_{a}^{C \gamma} D^{\alpha} x_{1}(t)\right)\right)= & h\left(t, x_{0}(t),{ }_{a}^{C \gamma} D^{\alpha} x_{0}(t)\right)-h\left(t, x_{1}(t),{ }_{a}^{C \gamma} D^{\alpha} x_{1}(t)\right) \\
& +h\left(t, x_{1}(t),{ }_{a}^{C \gamma} D^{\alpha} x_{1}(t)\right)-\lambda\left[\phi_{p}\left({ }_{a}^{C \gamma} D^{\alpha} x_{1}(t)\right)-\phi_{p}\left({ }_{a}^{C \gamma} D^{\alpha} x_{0}(t)\right)\right] \\
\leq & -\lambda\left[\phi_{p}\left({ }_{a}^{C \gamma} D^{\alpha} x_{0}(t)\right)-\phi_{p}\left({ }_{a}^{C \gamma} D^{\alpha} x_{1}(t)\right)\right]+h\left(t, x_{1}(t),{ }_{a}^{C \gamma} D^{\alpha} x_{1}(t)\right) \\
& -\lambda\left[\phi_{p}\left({ }_{a}^{C \gamma} D^{\alpha} x_{1}(t)\right)-\phi_{p}\left({ }_{a}^{C \gamma} D^{\alpha} x_{0}(t)\right)\right]=h\left(t, x_{1}(t),{ }_{a}^{C \gamma} D^{\alpha} x_{1}(t)\right), \\
{ }_{a} T^{\alpha} \phi_{p}\left({ }_{a}^{C \gamma} D^{\alpha} x_{1}(a)\right)= & b_{k}, \\
x_{1}(a)= & \int_{a}^{b}\left[w\left(s, x_{0}(s)\right)-w\left(s, x_{1}(s)\right)+w\left(s, x_{1}(s)\right)+\eta\left(x_{1}(s)-x_{0}(s)\right)\right] \mathrm{d} s+\rho \\
\leq & \int_{a}^{b}\left[\eta\left(x_{0}(s)-x_{1}(s)\right)+\eta\left(x_{1}(s)-x_{0}(s)\right)+w\left(s, x_{1}(s)\right)\right] \mathrm{d} s+\rho \\
= & \int_{a}^{b} w\left(s, x_{1}(s)\right) \mathrm{d} s+\rho .
\end{aligned}
$$

Clearly, $x_{1}(t)$ is a lower solution of (9). Similarly, $y_{1}(t)$ is an upper solution of (9). We obtain by applying mathematical induction that

$$
\begin{aligned}
x_{0}(t) & \leq x_{1}(t) \leq \cdots \leq x_{n}(t) \leq \cdots \leq y_{n}(t) \leq \cdots \leq y_{1}(t) \leq y_{0}(t), \\
{ }_{a}^{C \gamma} D^{\alpha} x_{0}(t){ }_{a}^{C \gamma} D^{\alpha} x_{1}(t) & \leq \cdots \leq{ }_{a}^{C \gamma} D^{\alpha} x_{n}(t) \leq \cdots{ }_{a}^{C \gamma} D^{\alpha} y_{n}(t) \\
& \leq \cdots \leq{ }_{a}^{C \gamma} D^{\alpha} y_{1}(t) \leq{ }_{a}^{C \gamma} D^{\alpha} y_{0}(t) .
\end{aligned}
$$

Step 2. We conclude that the sequences $\left\{x_{n}\right\}$ and $\left\{y_{n}\right\}$ satisfy the relations:

$$
\begin{aligned}
\lim _{n \longrightarrow \infty} x_{n}(t) & =x^{*}(t), \\
\lim _{n \longrightarrow \infty}{ }_{a}^{C \gamma} D^{\alpha} x_{n}(t) & ={ }_{a}^{C \gamma} D^{\alpha} x^{*}(t), \\
\lim _{n \longrightarrow \infty} y_{n}(t) & =y^{*}(t), \\
\lim _{n \longrightarrow \infty}{ }_{a}^{C \gamma} D^{\alpha} y_{n}(t) & ={ }_{a}^{C \gamma} D^{\alpha} y^{*}(t) .
\end{aligned}
$$

$$
\begin{aligned}
& \left.x_{n}(t)\right) \int_{a}^{b} G(t, s) \phi_{q}\left[\sum_{j=0}^{n-1} \frac{b_{j}(s-a)^{j \alpha}}{\alpha^{j}} E_{\beta, j+1}\left(\frac{-\lambda(s-a)^{\alpha \beta}}{\alpha^{\beta}}\right)\right. \\
& \left.+\int_{a}^{s} K^{\beta-1}(s, \theta) E_{\beta, \beta}\left(-\lambda K^{\beta}(s, \theta)\right) \frac{F\left(x_{n-1}(\theta)\right) \mathrm{d} \theta}{(\theta-a)^{1-\alpha}}\right] \frac{\mathrm{d} s}{(s-a)^{1-\alpha}} \\
& +\frac{\int_{a}^{b}\left[w\left(s, x_{n-1}(s)\right)-\eta x_{n-1}(s)\right] \mathrm{d} s+\rho}{1-\eta(b-a)} .
\end{aligned}
$$

Clearly, $\left\{x_{n}\right\}$ is uniformly bounded in $C_{\gamma}(J)$. By the continuity of $F, G, \phi_{q}$, and $K$, we can easily get that $\left\{x_{n}\right\}$ is equicontinuous. By the Arzelà-Ascoli theorem, we have that $\left\{x_{n}\right\}$ satisfies (69). In the same way, we get that $\left\{y_{n}\right\}$ satisfies (70). Moreover, $x^{*}(t)$ and $y^{*}(t)$ are solutions of (9).
Let $F\left(x_{n}\right)(t)=h\left(t, x_{n}(t),{ }_{a}^{C \gamma} D^{\alpha} x_{n}(t)\right)+\lambda \phi_{p}\left({ }_{a}^{C \gamma} D^{\alpha} x_{n}(t)\right)$. We can see that the function $F$ is continuous and nondecreasing from the assumption of $h$. By (37) and (54), equation (59) can be reduced to the equation
Step 3. We prove that $x^{*}$ and $y^{*}$ are extremal solutions of problem (9).

Assume that any solution $x(t)$ of problem (9) satisfies $x_{n}(t) \leq x(t) \leq y_{n}(t)$. Let $u(t)=\phi_{p}\left({ }_{a}^{C \gamma} D^{d} x(t)\right)-\phi_{p}$
$\left({ }_{a}^{C \gamma} D^{\alpha} x_{n+1}(t)\right)$, and by $\left(H_{3}\right)$, we have 


$$
\begin{aligned}
{ }_{a}^{C \beta} D^{\alpha} u(t)= & h\left(t, x(t),{ }_{a}^{C \gamma} D^{\alpha} x(t)\right)-h\left(t, x_{n}(t),{ }_{a}^{C \gamma} D^{\alpha} x_{n}(t)\right) \\
& +\lambda\left[\phi_{p}\left({ }_{a}^{C \gamma} D^{\alpha} x_{n+1}(t)\right)-\phi_{p}\left({ }_{a}^{C \gamma} D^{\alpha} x_{n}(t)\right)\right] \\
\geq & -\lambda u(t), \\
{ }_{a}^{k} T^{\alpha} u(a)= & 0 .
\end{aligned}
$$

From Lemma 7 , we have $u(t) \geq 0$, i.e., $\phi_{p}\left({ }_{a}^{C \gamma} D^{\alpha} x(t)\right) \geq \phi_{p}\left({ }_{a}^{C \gamma} D^{\alpha} x_{n+1}(t)\right)$. Hence,

$$
{ }_{a}^{C \gamma} D^{\alpha} x(t) \geq{ }_{a}^{C \gamma} D^{\alpha} x_{n+1}(t) .
$$

Let $\widetilde{u}(t)=x(t)-x_{n+1}(t)$, and by $\left(H_{4}\right),(59)$, and (73), we have

$$
\left\{\begin{array}{l}
{ }_{a}^{C \gamma} D^{\alpha} \widetilde{u}(t) \geq 0 \\
\widetilde{u}(a)=\int_{a}^{b}\left[w(s, x(s))-w\left(s, x_{n}(s)\right)-\eta\left(x_{n+1}(s)-x_{n}(s)\right)\right] \mathrm{d} s \geq \eta \int_{a}^{b} \widetilde{u}(s) \mathrm{d} s .
\end{array}\right.
$$

We get $\widetilde{u}(t) \geq 0$ from Lemma 8 , i.e., $x(t) \geq x_{m+1}(t)$. Similarly, ${ }_{a}^{C \gamma} D^{\alpha} y_{n+1}(t) \geq{ }_{a}^{C \gamma} D^{\alpha} x(t), y_{n+1}(t) \geq x(t)$. Hence, $x_{n+1}(t) \leq x(t) \leq y_{n+1}(t)$ holds. Therefore, $x^{*}(t) \leq x(t) \leq$ $y^{*}(t)$ as $n \longrightarrow \infty, \forall t \in J$. This ends the proof.

Remark 5. In [37], the authors assume that $h \in C([0,1] \times t[0,+\infty) n \times q(-\infty, 0] h,[0,+\infty))$, $h\left(t, w_{1}, z_{1}\right) \leq h\left(t, w_{2}, z_{2}\right) \quad$ for $\quad 0 \leq w_{1}<w_{2}, z_{1}>z_{2} \geq 0$, $t \in[0,1]$. The nonlinear term $h$ in this paper satisfies the weaker conditions.

\section{Example}

We present a numerical example as follows:

$$
\left\{\begin{array}{l}
{ }_{0}^{(1 / 2)} D^{(1 / 2)} \phi_{2}\left({ }_{0}^{(1 / 2)} D^{(1 / 2)} x(t)\right)=t^{(3 / 4)}+\frac{x(t)}{3\left(t^{(1 / 4)}+t^{-(3 / 4)}\right)}+\frac{1}{4^{0}}{ }^{(1 / 2)} D^{(1 / 2)} x(t), \quad t \in[0,1], \\
\phi_{2}\left({ }_{0}^{(1 / 2)} D^{(1 / 2)} x(0)\right)=0, \\
x(0)=\int_{0}^{1}\left[\frac{1}{7}(s+1) x(s)+s\right] \mathrm{d} s+1,
\end{array}\right.
$$

where $k=0, b_{0}=0, p=2, a=0, b=1, \rho=1, \beta=\gamma=\alpha=$

$(1 / 2)$ and

$$
\left\{\begin{array}{l}
h\left(t, x(t),{ }_{a}^{(1 / 2)} D^{(1 / 2)} x(t)\right)=t^{(3 / 4)}+\frac{x(t)}{3\left(t^{(1 / 4)}+t^{-(3 / 4)}\right)}+\frac{1}{4^{0}}{ }^{0} D^{(1 / 2)} x(t), \\
w(t, x(t))=\frac{1}{7}(t+1) x(t)+t .
\end{array}\right.
$$


Taking $x_{0}(t)=0, y_{0}(t)=3 t+3$, we can get

$$
\begin{aligned}
& \begin{cases}{ }_{0}^{(1 / 2)} D^{(1 / 2)} \phi_{2}\left({ }_{0}^{(1 / 2)} D^{(1 / 2)} x_{0}(t)\right)=0 \leq t^{(3 / 4)}+\frac{x(t)}{3\left(t^{(1 / 4)}+t^{-(3 / 4)}\right)}+\frac{1}{4^{0}}{ }^{(1 / 2)} D^{(1 / 2)} x_{0}(t), & t \in[0,1], \\
\phi_{2}\left({ }_{0}^{(1 / 2)} D^{(1 / 2)} x_{0}(0)\right)=0, & x_{0}(0)<\frac{3}{2},\end{cases} \\
& \begin{cases}{ }_{0}^{(1 / 2)} D^{(1 / 2)} \phi_{2}\left({ }_{0}^{(1 / 2)} D^{(1 / 2)} y_{0}(t)\right)={ }_{0}^{(1 / 2)} D^{(1 / 2)}\left(\frac{4 \sqrt{2}}{\sqrt{\pi}} t^{(3 / 4)}\right)=3 t^{(1 / 2)} \geq\left(2+\frac{\sqrt{2}}{\sqrt{\pi}}\right) t^{(3 / 4)}, & t \in[0,1], \\
\phi_{2}\left({ }_{0}^{(1 / 2)} D^{(1 / 2)} y_{0}(0)\right)=0, & y_{0}(0)=3>\frac{5}{2} .\end{cases}
\end{aligned}
$$

Hence, $x_{0}$ and $y_{0}$ are lower and upper solutions of (75), respectively. Therefore, $\left(\mathrm{H}_{2}\right)$ is satisfied. For $x_{0}(t) \leq x \leq$ $y \leq y_{0}(t)$

$$
\begin{aligned}
& h\left(t, y(t),{ }_{0}^{(1 / 2)} D^{(1 / 2)} y(t)\right)-h\left(t, x(t),{ }_{0}^{(1 / 2)} D^{(1 / 2)} x(t)\right) \\
& =\frac{y(t)-x(t)}{3\left(t^{(1 / 4)}+t^{-(3 / 4)}\right)}+\frac{1}{4^{0}}{ }^{(1 / 2)} D^{(1 / 2)}(y(t)-x(t)) \\
& \geq \frac{1}{4^{0}} D^{(1 / 2)} D^{(1 / 2)}(y(t)-x(t)) \\
& =\frac{1}{4}\left[\phi_{2}\left({ }_{0}^{(1 / 2)} D^{(1 / 2)} y(t)\right)-\phi_{2}\left({ }_{0}^{(1 / 2)} D^{(1 / 2)} x(t)\right)\right], \\
& \\
& w(t, y(t))-w(t, x(t)) \geq \frac{1}{7}(y(t)-x(t)) .
\end{aligned}
$$

We can see that $\lambda=-(1 / 4)<0, \eta=(1 / 7)$. Therefore, $\left(H_{3}\right)$ and $\left(H_{4}\right)$ hold. In light of Theorem 1 , the extremal solutions of $(75)$ can be obtained in $\left[x_{0}, y_{0}\right]$.

\section{Conclusions}

In this paper, we mainly use the montone iterative technique to study the Caputo conformable differential equations with $p$-Laplacian operator and integral boundary condition. A minimal and a maximal solution between the lower and the upper solutions are obtained. This method provides a constructive procedure for the solutions, and it is also useful for the investigation of qualitative properties of solutions. Since the Caputo conformable derivative can be reduced to the traditional Caputo derivative, some results produced from the traditional Caputo differential system can be seen as special cases of this paper. Moreover, the Caputo conformable derivative depends on two parameters naturally and the one coming from conformable operator can better describe long-memory processes. We believe that the Caputo and RL conformable fractional operators will play a key role in studying new types of fractional variational problems, Sturm-Liouville problems, optimal control problems, and modeling of complex systems.

\section{Abbreviations}

RL: Riemann-Liouville

BVP: Boundary value condition.

\section{Data Availability}

No data were used to support this study.

\section{Conflicts of Interest}

The authors declare that they have no conflicts of interest. 


\section{Authors' Contributions}

All authors contributed equally to this study and read and approved the final manuscript.

\section{Acknowledgments}

We would like to thank Professor Desheng Li for many helpful conversations. This study was supported by the National Natural Science and Mathematics Foundation of China (grant no. 11526177).

\section{References}

[1] A. A. Kilbas, H. M. Srivastava, and J. J. Trujillo, Theory and Applications of Fractional Differential Equatuins, Elsevier, Amsterdam, Netherlands, 2006.

[2] I. Podlubny, Fractional Differential Equations, Academic Press, New York, NY, USA, 1999.

[3] K. S. Miller and B. Ross, An Introduction to the Fractional Calculus and Fractional Differential Equation, Wiley, New York, NY, USA, 1993.

[4] R. Hilfer, Applications of Fractional Calculus in Physics, Word Scientific, Singapore, 2015.

[5] J. T. Machado, V. Kiryakova, and F. Mainardi, "Recent history of fractional calculus," Communications in Nonlinear Science and Numerical Simulation, vol. 16, no. 3, pp. 1140-1153, 2011.

[6] C. F. Lorenzo and T. T. Hartley, "Variable order and distributed order fractional operators," Nonlinear Dynamics, vol. 29, no. 1, pp. 57-98, 2002.

[7] B. Ahmad, A. Alsaedi, S. K. Ntouyas, and J. Tariboon, Hadamard-Type Fractional Differential Equations, Inclusions and Inequalities, Springer, Berlin, Germany, 2017.

[8] S. G. Samko, A. A. Kilbas, and O. I. Marichev, Fractional Integrals and Derivatives: Theory and Applications, Gordon \& Breach, Yverdon, Switzerland, 1993.

[9] F. Jarad, T. Abdeljawad, and D. Baleanu, "Caputo-type modification of the hadamard fractional derivatives," Advances in Difference Equations, vol. 2012, no. 1, p. 142, 2012.

[10] R. Khalil, M. Al Horani, A. Yousef, and M. Sababheh, "A new definition of fractional derivative," Journal of Computational and Applied Mathematics, vol. 264, pp. 65-70, 2014.

[11] T. Abdeljawad, "On conformable fractional calculus," Journal of Computational and Applied Mathematics, vol. 279, pp. 57-66, 2015.

[12] F. Jarad, E. Uğurlu, T. Abdeljawad, and D. Baleanu, "On a new class of fractional operators," Advances in Difference Equations, vol. 2017, no. 1, p. 247, 2017.

[13] T. Abdeljawad, Q. M. Al-Mdallal, and F. Jarad, "Fractional logistic models in the frame of fractional operators generated by conformable derivatives," Chaos, Solitons \& Fractals, vol. 119, pp. 94-101, 2019.

[14] T. Abdeljawad, "Fractional operators with boundary points dependent kernels and integration by parts," Discrete \& Continuous Dynamical Systems - S, vol. 13, no. 3, pp. 351-375, 2020.

[15] G. S. Ladde, V. Lakshmikantham, and A. S. Vatsala, Monotone Iterative Technique for Nonlinear Differential Equations, Pitman, Boston, MA, USA, 1985.

[16] H.-K. Xu and J. J. Nieto, "Extremal solutions of a class of nonlinear integro-differential equations in banach spaces," Proceedings of the American Mathematical Society, vol. 125, no. 9, pp. 2605-2614, 1997.
[17] B. Ahmad and J. J. Nieto, "The monotone iterative technique for three-point second-order integrodifferential boundary value problems with p-laplacian," Boundary Value Problems, vol. 2007, no. 7, pp. 1-9, 2007.

[18] J. J. Nieto and R. Rodríguez-López, "New comparison results for impulsive integro-differential equations and applications," Journal of Mathematical Analysis and Applications, vol. 328, no. 2, pp. 1343-1368, 2007.

[19] W. Wang, J. Shen, and Z. Luo, "Multi-point boundary value problems for second-order functional differential equations," Computers \& Mathematics with Applications, vol. 56, no. 8, pp. 2065-2072, 2008.

[20] J. J. Nieto and N. Alvarez-Noriega, "Periodic boundary value problems for nonlinear first order ordinary differential equations," Acta Mathematica Hungarica, vol. 71, no. 1-2, pp. 49-58, 1996.

[21] Z. Luo and J. J. Nieto, "New results for the periodic boundary value problem for impulsive integro-differential equations," Nonlinear Analysis: Theory, Methods \& Applications, vol. 70, no. 6, pp. 2248-2260, 2009.

[22] Z. He and J. Yu, "Periodic boundary value problem for firstorder impulsive ordinary differential equations," Journal of Mathematical Analysis and Applications, vol. 272, no. 1, pp. 67-78, 2002.

[23] Z. Luo and Z. Jing, "Periodic boundary value problem for first-order impulsive functional differential equations," Computers \& Mathematics with Applications, vol. 55, no. 9, pp. 2094-2107, 2008.

[24] J. J. Nieto and R. Rodríguez-López, "Periodic boundary value problem for non-lipschitzian impulsive functional differential equations," Journal of Mathematical Analysis and Applications, vol. 318, no. 2, pp. 593-610, 2006.

[25] W. Ding, Y. Xing, and M. Han, "Anti-periodic boundary value problems for first order impulsive functional differential equations," Applied Mathematics and Computation, vol. 186, no. 1, pp. 45-53, 2007.

[26] M. Yao, A. Zhao, and J. Yan, "Anti-periodic boundary value problems of second order impulsive differential equations," Computers \& Mathematics with Applications, vol. 59, no. 12, pp. 3617-3629, 2010.

[27] Z. Luo, J. Shen, and J. J. Nieto, "Antiperiodic boundary value problem for first-order impulsive ordinary differential equations," Computers \& Mathematics with Applications, vol. 49, no. 2-3, pp. 253-261, 2005.

[28] T. Jankowski, "Positive solutions for second order impulsive differential equations involving stieltjes integral conditions," Nonlinear Analysis: Theory, Methods \& Applications, vol. 74, no. 11, pp. 3775-3785, 2011.

[29] G. Song, Y. Zhao, and X. Sun, "Integral boundary value problems for first order impulsive integro-differential equations of mixed type," Journal of Computational and Applied Mathematics, vol. 235, no. 9, pp. 2928-2935, 2011.

[30] S. Zhang, "Monotone iterative method for initial value problem involving Riemann-Liouville fractional derivatives," Nonlinear Analysis: Theory, Methods \& Applications, vol. 71, no. 5-6, pp. 2087-2093, 2009.

[31] Z. Wei, W. Dong, and J. Che, "Periodic boundary value problems for fractional differential equations involving a Riemann-Liouville fractional derivative," Nonlinear Analysis: Theory, Methods \& Applications, vol. 73, no. 10, pp. 3232$3238,2010$.

[32] T. Jankowski, "Boundary problems for fractional differential equations," Applied Mathematics Letters, vol. 28, pp. 14-19, 2014. 
[33] L. Zhang, B. Ahmad, and G. Wang, "The existence of an extremal solution to a nonlinear system with the right-handed Riemann-Liouville fractional derivative," Applied Mathematics Letters, vol. 31, pp. 1-6, 2014.

[34] G. Wang, "Monotone iterative technique for boundary value problems of a nonlinear fractional differential equation with deviating arguments," Journal of Computational and Applied Mathematics, vol. 236, no. 9, pp. 2425-2430, 2012.

[35] L. Zhang, B. Ahmad, and G. Wang, "Explicit iterations and extremal solutions for fractional differential equations with nonlinear integral boundary conditions," Applied Mathematics and Computation, vol. 268, pp. 388-392, 2015.

[36] L. S. Lejbenson, "General problem of the movement of a compressible fluid in a porous medium," Izv Akad Nauk SSSR, Ser Geogr Geofiz.vol. 9, pp. 7-10, 1983.

[37] X. Liu, M. Jia, and W. Ge, "The method of lower and upper solutions for mixed fractional four-point boundary value problem with p-Laplacian operator," Applied Mathematics Letters, vol. 65, pp. 56-62, 2017.

[38] J. Qin, G. Wang, L. Zhang, and B. Ahmad, "Monotone iterative method for a p-Laplacian boundary value problem with fractional conformable derivatives," Boundary Value Problems, vol. 2019, no. 1, p. 145, 2019.

[39] Y. Liu and X. Yang, "Resonant boundary value problems for singular multi-term fractional differential equations," Differential Equations \& Applications, vol. 5, no. 3, pp. 409-472, 2013. 